\section{A quantitative analysis of the relation between the clavicular tilt angle and subclavian central venous catheter misplacement}

\author{
Hoe-Hwan Jeong ${ }^{1}$, Jung-Hoon Yoon ${ }^{1}$, Sungho $\mathrm{Oh}^{2}$, Je Hwan Won ${ }^{3}$, \\ Young-Gi Min ${ }^{1}$, Nikolaus Gravenstein ${ }^{4}$, Sang-Cheon Choi ${ }^{1}$ \\ 'Department of Emergency Medicine, Ajou University School of Medicine, Suwon, Korea \\ ${ }^{2}$ Daegu Research Center for Medical Device and Green Energy, Korea Institute of Machinery \& Materials, \\ Daegu, Korea \\ ${ }^{3}$ Department of Radiology, Ajou University School of Medicine, Suwon, Korea \\ ${ }^{4}$ Department of Anesthesiology, University of Florida College of Medicine, Gainesville, FL, USA
}

Objective The aim of the present study was to investigate the relation between shoulder position and subclavian central venous (SCV) catheter misplacement. The shoulder position was estimated using clavicular tilt angle (CTA) values observed on anteroposterior chest X-ray images.

Methods A retrospective case-control study was conducted on all adult patients who underwent SCV catheterization in the emergency department during a 12-month period. Collected data included patient age, sex, diagnosis, catheterization side, catheter misplacement, and physician's level of experience in catheterization. The CTA and other radiological variables such as the ipsilateral transverse length of the thorax and thickness of the clavicle were investigated.

Results Among all central venous catheterizations $(n=1,599)$, the subclavian route was used 981 times (61.4\%). There were 51 misplacements of SCV catheters (5.2\%) during the study period. There were no differences in the sex, age, blood pressure, and diagnosis between the two groups. The CTA values were $28.5^{\circ} \pm 7.3^{\circ}$ and $22.6^{\circ} \pm 6.3^{\circ}$ in the misplacement group and control group, respectively ( $95 \%$ confidence interval, 3.6 to $8.1 ; \mathrm{P}<0.001)$.

Conclusion In this study, the CTA was found to be $5.9^{\circ}$ larger in the misplacement group than in the control group. Assuming that CTA indicates the shoulder position, our findings suggest that the chance of SCV catheter misplacement may be reduced by avoiding the shoulder elevated.

Keywords Subclavian vein; Catheterization; Treatment failure; Radiography; Clavicle

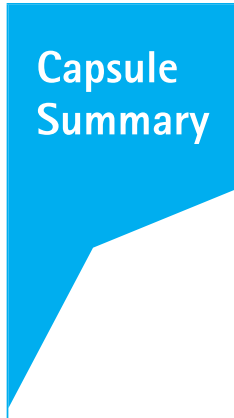

What is already known

There is no accepted method to quantify shoulder position, although there are several previous studies regarding the optimal shoulder position during subclavian vein catheterization.

What is new in the current study

Assuming that clavicular tilt angle reflects shoulder position, our findings suggest that one can reduce the risk of subclavian central venous catheters misplacement by avoiding shoulder elevation.
elSSN: 2383-4625

Received: 20 June 2014

Revised: 1 August 2014

Accepted: 8 August 2014

Correspondence to: Sang-Cheon Choi Department of Emergency Medicine, Ajou University School of Medicine, 206 World cup-ro, Yeongtong-gu,

Suwon 443-721, Korea

E-mail: avenue59@ajou.ac.kr

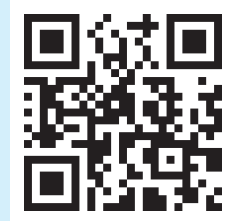

How to cite this article:

Jeong $\mathrm{HH}$, Yoon JH, Oh S, Won JH, Min YG, Gravenstein N, Choi SC. A quantitative analysis of the relation between the clavicular tilt angle and subclavian central venous catheter misplacement. Clin Exp Emerg Med 2014;1(2):114-119.

This is an Open Access article distributed under the terms of the Creative Commons Attribution Non-Commercial License (http:// creativecommons.org/licenses/by-nc/3.0/). 


\section{INTRODUCTION}

Catheter tip malpositioning into other central veins may increase the risk of chemical or bacterial venous thrombophlebitis and vascular perforation. ${ }^{1}$ In addition, accurate central venous pressure monitoring will be impossible. ${ }^{2-4}$ When a subclavian central venous (SCV) catheter is misplaced into the ipsilateral internal jugular vein (IJV), catastrophic events such as encephalopathy or infection may occur due to elevated intracranial pressure or thrombosis associated with the misplacement. ${ }^{4-7}$

Compared to the IJV route, the SCV route has a greater chance of misplacement. ${ }^{8-10}$ To decrease the occurrence rates of SCV catheter misplacement, using the Ambesh maneuver to orient the needle bevel and shoulder and head positions are considered effective to a certain degree., ${ }^{2,11,12}$ Still, the reported incidence of unintentional positioning of a central venous catheter tip in other central veins ranges from $2 \%$ to $21 \% .^{11-14}$ Confirmation of the misplacement could be made using bedside techniques such as saline flush testing, pressure monitoring, intravascular electrocardiography, and ultrasonographic confirmation. ${ }^{1,15-18}$ Use of these methods is largely limited as they are not routine methods in emergencies; therefore, it is important to prevent catheter misplacement.

Previous studies have reported comparisons between misplacement rates and patients' shoulder positions. ${ }^{19-21} \mathrm{~A}$ recent randomized controlled trial suggested that the neutral position was associated with a reduced misplacement rate, but the preferred shoulder position (lowered, elevated, or neutral) for misplacement prevention remains controversial. ${ }^{20}$

To date, there has been no quantitative evaluation of shoulder position in the context of catheter misplacement. We attempted to evaluate geometric variables such as the clavicular tilt angle (CTA) obtained from anteroposterior (AP) chest $\mathrm{X}$-ray images to determine their correlations with patients' shoulder positions and investigate the relation of such variables with the chance of catheter misplacement. Although limited, our study introduced a quantitative method for and insight into investigating SCV catheter misplacement, a potential problem plaguing emergency physicians. ${ }^{22,23}$ The present study was conducted to investigate the relation between shoulder position and SCV catheter misplacement. Shoulder position was estimated using CTA values observed on AP chest $X$-ray images.

\section{METHODS}

\section{Study design and setting}

A retrospective case-control study was conducted at a 1,080-bed academic tertiary referral center. The annual emergency depart- ment (ED) volume is 90,000 visits, including 70\% adult visits. The hospital has been using electronic medical records and picture archiving and communication system (PACS). The present study was approved by the hospital's Institutional Review Board, and informed consent was waived.

\section{Selection of participants}

The study was conducted on all the adult patients ( $>18$ years of age) who underwent central venous catheterization in the ED of our hospital between January and December 2012. We closely investigated cases of SCV catheter misplacement. Catheterization was performed using double/triple lumen non-tunneled infusion catheters (ARROWguard, Blue PLUS central venous catheter, Arrow International Inc., Reading, PA, USA). Exclusion criteria were (1) patients who underwent catheterization via the Ambesh maneuver; (2) those who had tunneled dialysis catheters; and (3) those who had rib or clavicle fractures.

All central venous catheterizations were conducted using a modified Seldinger technique without ultrasonographic guidance at the patients' bedside. The catheterization procedure applied during the study period was as follows: (1) Place the patient in a supine position and the patient's head in a neutral position. (2) Clean the neck and infraclavicular areas including the suprasternal notch with $0.5 \%$ chlorhexidine solution. Isolate them with sterile drapes. (3) Anesthetize the region by applying 1\% lidocaine around the midpoint of the clavicle. (4) Place the index finger and the thumb of the non-dominant hand on the suprasternal notch and the medial two-thirds of the clavicle, respectively. Find the puncture point directly below, where the lateral third of the clavicle meets the medial two-thirds. (5) Direct the introducer needle with the bevel facing down towards the suprasternal notch at a $10^{\circ}$ angle to the surface of the chest. (6) Advance the $18 \mathrm{G}$ introducer needle slowly through the skin and subcutaneous tissues until flush blood or dark venous blood appears. Seek help from a more experienced physician after three failed attempts. (7) Check for continual free venous flow with aspiration. (8) Insert a J-tip guidewire while maintaining a caudal direction and remove the introducer needle. (9) Dilate the skin and subcutaneous tissues overlying the guidewire using a dilator. (10) Railroad a central catheter over the guidewire $12-14 \mathrm{~cm}$ into the subclavian vein. (11) Remove the guidewire. (12) Confirm catheter functions and suture the catheter. (13) Apply a sterile transparent dressing. (14) Confirm correct catheter placement on a chest X-ray image.

In our study, experienced physicians were those who performed the above catheterization procedure 50 or more times..$^{24,25}$ For every patient with a misplaced catheter, two other patients who underwent catheterization within 30 days of the time when the 
misplacement occurred were matched as the control group under two conditions: (1) they were the same sex as the misplacement case, and (2) the difference in ages was minimum.

\section{Data collection and processing}

Demographic, clinical, and radiological data were extracted from patient medical records and the AP chest X-ray image database in a standardized fashion. We performed a 1:2 matched casecontrol study to constrain possible confounding factors. We randomly selected $20 \%$ of the records for an independent review by an abstractor. Discrepancies arising from the review of the results by other data abstractors were resolved with the help of a radiologist or a senior emergency physician. The collected data included age, sex, diagnosis, catheterization side, physician's level of experience, and the occurrence of catheter misplacement. Catheter misplacement was confirmed on AP chest X-ray images by examining the catheter tip. The intended position was between the SCV and the right atrium. Malpositioning was typically found in the ipsilateral IJV, contralateral brachiocephalic vein (BCV), or

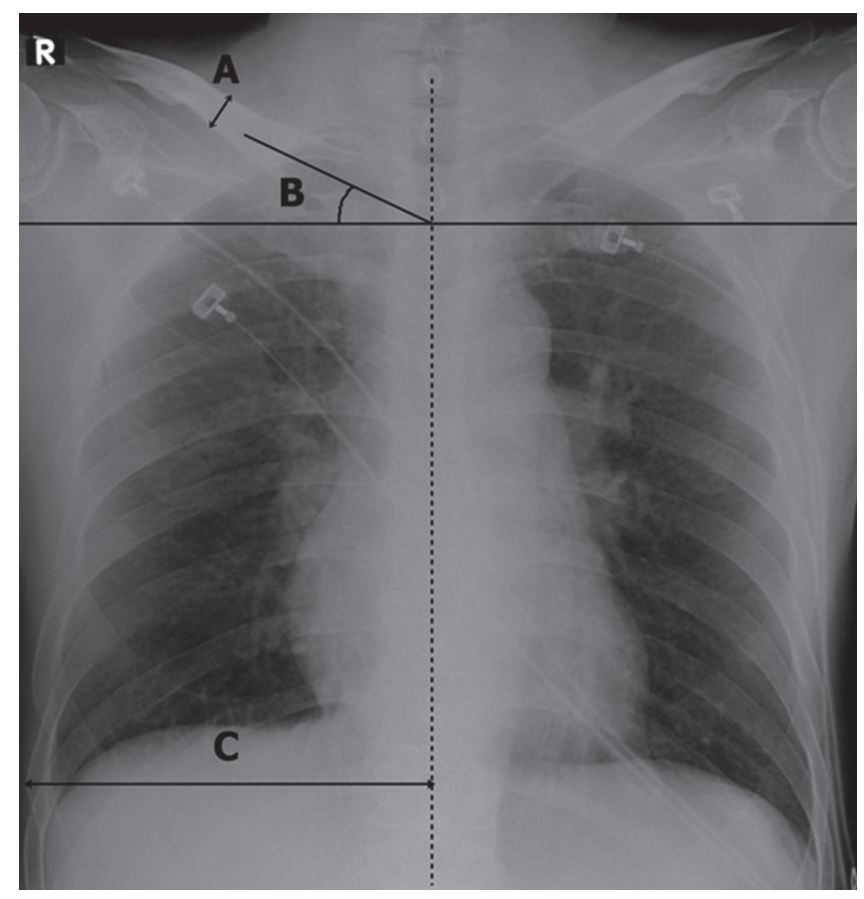

Fig. 1. Chest radiography definitions. (A) Thickness of the clavicle. (B) Clavicular tilt angle. (C) Ipsilateral transverse length of the thorax. Thickness of the clavicle was defined as the superior-inferior length at the midpoint between the sternal end and acromial end of the clavicle. The clavicular tilt angle was defined as the angle between two vectors on an anteroposterior chest X-ray image: (1) the line bisecting the proximal portion of the clavicle and (2) the horizontal line. The ipsilateral transverse length of the thorax was defined as the longest transverse length between the spinous process of the thoracic vertebrae and the most laterally located rib on radiographic images. contralateral IJV.

Geometric variables were estimated from the X-ray images, and they included the ipsilateral transverse length of the thorax (ITL-T), CTA, and thickness of the clavicle (TC). TC was defined as the superior-inferior width at the midpoint between the sternal end and acromial end of the clavicle. The CTA was defined as the angle between two vectors, i.e., the line bisecting the proximal portion of the clavicle and the horizontal line. ${ }^{22,24}$ CTA is a geometric variable for evaluating shoulder balance/imbalance. ${ }^{22,23}$ The ITL-T was defined as the longest transverse length between the spinous process of the thoracic vertebrae and the most laterally located rib shadow. In Fig. 1, these variables are displayed on a chest X-ray image. Geometric measurement values were entered into the software analysis as tabulation (Excel 2013, Microsoft Co., Redmond, WA, USA).

\section{Primary data analysis}

Inter-rater reliability was assessed using the generalized K-statistic and 95\% confidence interval $(\mathrm{Cl})$ for four data elements: catheter misplacement and appropriateness of CTA, TC, and ITL-T. All data were expressed as mean $\pm S D$ as appropriate, and they were analyzed using a statistical tool (Stata IC ver. 11, Stata Co., College Station, TX, USA). The significance of inter-group differences was assessed with the chi-square test for categorical variables and the t-test for continuous variables. A two-sided P-value less than 0.05 was considered to indicate statistical significance.

\section{RESULTS}

A total of 1,818 patients underwent central venous catheterization during our study period, and 1,599 cases were considered for inclusion (Fig. 2). Of these catheterizations, the subclavian route was used 981 times (61.4\%) and misplacement occurred 51 times (5.2\%). Misplacement into the ipsilateral IJV occurred 43 times (right, 41; left, 2) and misplacement into the contralateral BCV occurred 8 times (right, 8; left, 0). No misplacement into the contralateral IJV occurred. Sixteen of the misplacements were by inexperienced physicians (less than 50 performances) and 35 were by experienced physicians (more than 50 performances).

Table 1 shows patient demographics and AP chest X-ray evaluation results. TC values in the misplacement group and the control group were $13.2 \pm 2.1 \mathrm{~mm}$ and $13.7 \pm 2.8 \mathrm{~mm}$, respectively $\left(95 \% \mathrm{Cl}_{1}-1.38\right.$ to $\left.0.35 ; \mathrm{P}=0.20\right)$. CTA values in the misplacement group and the control group were $28.5^{\circ} \pm 7.3^{\circ}$ and $22.6^{\circ} \pm 6.3^{\circ}$, respectively. The CTA value in the misplacement group was significantly greater than that in the control group $(95 \% \mathrm{Cl}, 3.63$ to $8.11 ; P<0.001)$. ITL-T values in the misplacement group and the 


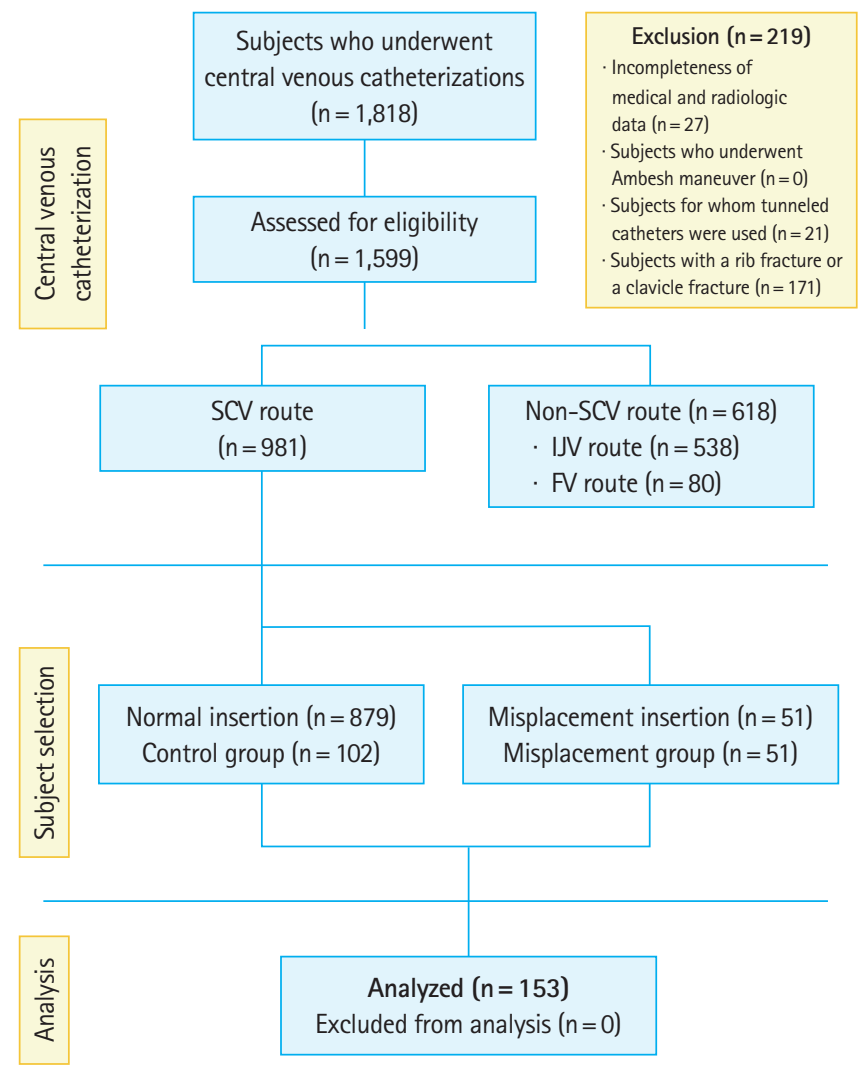

Fig. 2. Flow chart of subject selection. SCV, subclavian vein; IJV, internal jugular vein; $\mathrm{FV}$, femoral vein.

control group were $137.0 \pm 11.6 \mathrm{~mm}$ and $138.7 \pm 13.7 \mathrm{~mm}$, respectively ( $95 \% \mathrm{Cl},-6.12$ to $2.71 ; \mathrm{P}=0.45)$.

The four data abstractors in our study reached agreement for more than $95 \%$ of the data elements. The value of Cohen $\mathrm{K}$ - was 0.99 for catheter misplacement $(95 \% \mathrm{Cl}, 0.96$ to 1.00$), 0.80$ for TC (95\% Cl, 0.67 to 0.94$), 0.89$ for CTA ( $95 \% \mathrm{Cl}, 0.72$ to 0.95$)$, and 0.94 for ITL-T ( $95 \% \mathrm{Cl}, 0.77$ to 1.0$)$.

\section{DISCUSSION}

Catheter insertion through the SCV is frequently performed in the $E D$, and catheter tip malpositioning should be avoided for improving patient prognosis. Previous studies of SCV catheter insertion mostly focused on factors associated with successful puncture of the SCV, catheterization failure, and positions of the malpositioned catheter tips along with the associated complications. $1,4,8,19,24,25$ In the present study, we attempted to associate incidences of misplacement with radiographic features of patient shoulders. The incidence of catheter misplacement in our study was $5.2 \%$, which falls in the range of previously reported values: $21.4 \%$ by Tripathi et al. ${ }^{12} 5.4 \%$ by Sanchez et al., ${ }_{13}^{13}$ and $2.1 \%$ by Ambesh et al. ${ }^{14}$ Inconsistencies of these percentage values must
Table 1. Comparison between the misplacement group and the control group

\begin{tabular}{lccc}
\hline Variable & $\begin{array}{c}\text { Misplacement } \\
\text { group }(\mathrm{n}=51)\end{array}$ & $\begin{array}{c}\text { Control group } \\
(\mathrm{n}=102)\end{array}$ & P-value \\
\hline Age (yr) & & & $0.78^{\text {a) }}$ \\
$\quad$ Median (interquartile range) & $70(54-76)$ & $68.5(54-76)$ & \\
$\quad$ Range & $28-86$ & $28-87$ & \\
Sex & & & $1.00^{\text {b) }}$ \\
$\quad$ Male & $29(56.9)$ & $58(56.9)$ & \\
Female & $22(43.1)$ & $44(43.1)$ & \\
Diagnosis & & & \\
Respiratory & $7(13.7)$ & $15(14.7)$ & \\
Hepatobiliary & $10(19.6)$ & $19(18.6)$ & \\
Cardiovascular & $7(13.7)$ & $13(12.7)$ & \\
Gastrointestinal & $4(7.8)$ & $9(8.8)$ & \\
Urogenital & $7(13.7)$ & $13(12.7)$ & \\
Traumatic & $6(11.8)$ & $13(12.7)$ & \\
Central nervous & $4(7.8)$ & $9(8.8)$ & \\
$\quad$ Others & $6(11.8)$ & $11(10.8)$ & \\
Clavicle thickness (mm) & $13.23 \pm 2.09$ & $13.74 \pm 2.75$ & $0.20^{\text {a) }}$ \\
Clavicle tilt angle ( $\left.{ }^{\circ}\right)$ & $28.48 \pm 7.32$ & $22.61 \pm 6.25$ & $<0.001^{\text {a) }}$ \\
Thorax transveres length, & $137.02 \pm 11.61$ & $138.73 \pm 13.70$ & $0.45^{\text {a) }}$ \\
ipsilateral (mm) & & & \\
\hline
\end{tabular}

Values are presented as number (\%) or mean \pm SD unless otherwise indicated. ${ }^{a}$ The t-test was used for statistical analysis; ${ }^{\text {b}}$ The chi-square test was used for statistical analysis.

be associated with various confounding factors such as the direction of the J-tip and varying competency levels of the performers, to name a few. In our study, all catheterizations strictly followed the standard protocol described above. Heretofore, the methods suggested for successful SCV catheterization included caudal positioning of the guidewire tip, caudal positioning of the plunger end after puncture, Ambesh maneuver, head positioning, and inferior positioning of the shoulder. ${ }^{12-14,26,27}$ The Ambesh maneuver refers to a manual compression of the ipsilateral IJV at the time of guidewire insertion to prevent its progression into the ipsilateral IJV. ${ }^{11}$ However, these methods are not always successful.

A quantitative study of shoulder position based on computed tomography images in the context of catheter misplacement was performed by Kitagawa et al. ${ }^{19}$ They found that a superior-inferior change of $5 \mathrm{~cm}$ in shoulder height increased the overlapping area between the clavicle and SCV from 33.5\% to 40\%, increased the angle between the $\mathrm{SCV}$ and IJV from $89^{\circ}$ to $117^{\circ}$, and decreased the angle between the $\mathrm{SCV}$ and $\mathrm{BCV}$ from $114^{\circ}$ to $99^{\circ}$ in the neutral shoulder position. These results imply that the shoulder should be kept in the inferior position, which will lead to a maximal overlapping region between the clavicle and the SCV, and a successful puncture of the SCV. ${ }^{19}$ However, controversy regarding this issue remains since Baltalarli et al. ${ }^{21}$ reported that maintaining a superior shoulder position resulted in higher success rates of SCV catheterization, although a relatively more consistent relationship 
between the clavicle and the SCV could have been a confounder.

We noted a lack of studies that approached this problem quantitatively. In this study, we measured CTA values from AP chest Xray images as described in Fig. $1 A$, and a CTA increase increase is likely to be associated with an upward change of shoulder position, while a CTA decrease is likely associated with a downward change. Mean CTA measurements were $28.5^{\circ}$ and $22.6^{\circ}$ in the misplacement and control groups, respectively. The CTA in the misplacement group was $5.9^{\circ}$ greater than that in the control group, and the difference was significant. This result suggests that decreasing the CTA and maintaining an inferior shoulder position could be important for reducing the risk of misplacement in SCV catheterization. It seems that the CTA is determined not only by shoulder position, but potentially also by other factors such as ITL-T, AP length of thorax, clavicle length, height, and body weight. Thus, future studies should evaluate the effect of the AP length and the length of the clavicle on SCV catheterization.

This retrospective study has many limitations. First, different levels of procedure competency may have affected the incidence rates of misplacement. This may not have been a major confounding factor, however, as the occurrence of misplacement in the present study falls within a reasonable range (2\% to $21.4 \%$, as previously reported). ${ }^{12-14}$ In addition, in our hospital, all procedures strictly followed the standard protocol. Second, the chest $X$-ray images were obtained at a follow-up after the catheter insertion. Therefore, the CTA values may not precisely match the actual shoulder angles at the time of insertion. This may have played a limited role as a confounder, as the same conditions were imposed on both groups. Third, possible confounders include respiratory movements, the physical condition of patients at acute clinical stages, and unadjusted directional characteristics between AP and PA. Fourth, our study is largely limited by the fact that only a univariate analysis was included in the study design. Future studies should address these issues.

Positional characteristics of the clavicle and the scapula are thought to be major factors determining patient shoulder position. $^{22,23,28}$ In our study, CTA alone was found to be associated with a higher occurrence rate of misplacement. Although limited, our investigation incorporated the use of geometric measures based on X-ray images such as CTA values. We believe that such variables provide $E D$ clinicians with a useful quantitative insight into the issue of catheter misplacement.

In summary, radiological evaluation found that the CTA was larger in the misplacement group than in the control group. Assuming that CTA indicates the actual shoulder position, our findings suggest that SCV catheter misplacement should occur less frequently by avoiding patient shoulder elevation.

\section{CONFLICT OF INTEREST}

No potential conflict of interest relevant to this article was reported.

\section{REFERENCES}

1. Mansfield PF, Hohn DC, Fornage BD, Gregurich MA, Ota DM. Complications and failures of subclavian-vein catheterization. N Engl J Med 1994;331:1735-8.

2. Jung CW, Bahk JH, Kim MW, Lee KH, Ko H. Head position for facilitating the superior vena caval placement of catheters during right subclavian approach in children. Crit Care Med 2002;30:297-9.

3. Conces DJ Jr, Holden RW. Aberrant locations and complications in initial placement of subclavian vein catheters. Arch Surg 1984;119:293-5.

4. Boardman P, Hughes JP. Radiological evaluation and management of malfunctioning central venous catheters. Clin Radiol 1998;53:10-6.

5. Rath GP, Bithal PK, Toshniwal GR, Prabhakar H, Dash HH. Saline flush test for bedside detection of misplaced subclavian vein catheter into ipsilateral internal jugular vein. $\mathrm{Br} J$ Anaesth 2009;102:499-502.

6. Vesely TM. Central venous catheter tip position: a continuing controversy. J Vasc Interv Radiol 2003;14:527-34.

7. Rosen M, Latto IP, Shang N. Handbook of percutaneous central venous catheterisation. London: WB Saunders; 1981.

8. Pikwer A, Baath L, Davidson B, Perstoft I, Akeson J. The incidence and risk of central venous catheter malpositioning: a prospective cohort study in 1,619 patients. Anaesth Intensive Care 2008;36:30-7.

9. Tekin M, Ozbay B, Kati I, Arslan H. Misplacements of central venous catheters: internal jugular versus subclavian access in critical care patients. Eur J Gen Med 2007;4:123-7.

10. Ruesch S, Walder B, Tramer MR. Complications of central venous catheters: internal jugular versus subclavian access: a systematic review. Crit Care Med 2002;30:454-60.

11. Ambesh SP, Dubey PK, Matreja P, Tripathi M, Singh S. Manual occlusion of the internal jugular vein during subclavian vein catheterization: a maneuver to prevent misplacement of catheter into internal jugular vein. Anesthesiology 2002;97:528-9.

12. Tripathi M, Dubey PK, Ambesh SP. Direction of the J-tip of the guidewire, in seldinger technique, is a significant factor in misplacement of subclavian vein catheter: a randomized, controlled study. Anesth Analg 2005;100:21-4.

13. Sanchez R, Halck S, Walther-Larsen S, Heslet L. Misplacement 
of subclavian venous catheters: importance of head position and choice of puncture site. Br J Anaesth 1990;64:632-3.

14. Ambesh SP, Pandey JC, Dubey PK. Internal jugular vein occlusion test for rapid diagnosis of misplaced subclavian vein catheter into the internal jugular vein. Anesthesiology 2001;95: 1377-9.

15. Pandey JC, Dubey PK. A method for rapid clinical diagnosis of misplaced subclavian vein catheters. Anesth Analg 2000;90: 229.

16. Nolsoe C, Nielsen L, Karstrup S, Lauritsen K. Ultrasonically guided subclavian vein catheterization. Acta Radiol 1989;30 :108-9.

17. Denys BG, Uretsky BF, Reddy PS, Ruffner RJ, Sandhu JS, Breishlatt WM. An ultrasound method for safe and rapid central venous access. N Engl J Med 1991;324:566.

18. Maury E, Guglielminotti J, Alzieu M, Guidet B, Offenstadt G. Ultrasonic examination: an alternative to chest radiography after central venous catheter insertion? Am J Respir Crit Care Med 2001;164:403-5.

19. Kitagawa N, Oda M, Totoki T, et al. Proper shoulder position for subclavian venipuncture: a prospective randomized clinical trial and anatomical perspectives using multislice computed tomography. Anesthesiology 2004;101:1306-12.

20. Kang M, Ryu HG, Son IS, Bahk JH. Influence of shoulder position on central venous catheter tip location during infracla- vicular subclavian approach. Br J Anaesth 2011;106:344-7.

21. Baltalarli A, Adiguzel E, Gurses E, Coskun E. Subclavian vein cannulation in a different position. J Neurol Sci Turk 2000;17:3.

22. Akel I, Pekmezci M, Hayran M, et al. Evaluation of shoulder balance in the normal adolescent population and its correlation with radiological parameters. Eur Spine J 2008;17:348-54.

23. Ludewig PM, Behrens SA, Meyer SM, Spoden SM, Wilson LA.Three-dimensional clavicular motion during arm elevation: reliability and descriptive data. J Orthop Sports Phys Ther 2004; 34:140-9.

24. Muhm M, Sunder-Plassmann G, Apsner R, et al. Malposition of central venous catheters: incidence, management and preventive practices. Wien Klin Wochenschr 1997;109:400-5.

25. Sznajder JI, Zveibil FR, Bitterman H, Weiner P, Bursztein S. Central vein catheterization: failure and complication rates by three percutaneous approaches. Arch Intern Med 1986;146: 259-61.

26. McGee DC, Gould MK. Preventing complications of central venous catheterization. N Engl J Med 2003;348:1123-33.

27. Tan BK, Hong SW, Huang MH, Lee ST. Anatomic basis of safe percutaneous subclavian venous catheterization. J Trauma 2000; 48:82-6.

28. Culham $E$, Peat M. Functional anatomy of the shoulder complex. J Orthop Sports Phys Ther 1993;18:342-50. 